\title{
Recognition of the Cybersecurity State of IoT devices
}

\author{
M.E. Sukhoparov ${ }^{1}$, I.S. Lebedev ${ }^{1}$ \\ ${ }^{1}$ St. Petersburg Federal Research Center of the Russian Academy of Sciences (SPC RAS), 39, \\ 14th Line V.O., 199178, Saint-Petersburg, Russian Federation
}

\begin{abstract}
The identification of the cybersecurity (CS) state of Internet of things (IoT) devices determines the necessity to search for and improve approaches to detecting various threat types. The unification used in the mass development of IoT devices facilitates software and hardware modification to block certain built-in protective functions from the side of a potential intruder. A need arises to develop universal methods for identifying the cybersecurity state of devices using comprehensive approaches to analyzing data from internal and external information channels. The article presents an approach to identifying the cybersecurity of IoT devices based on processing time series recorded from sensors during various processes, and internal and external (thirdparty) sources. The approach is based on classification methods. The presented solution uses template sequences containing synchronized time series showing numerical values obtained from various probes and sensors during process execution. The proposed approach makes it possible to identify IoT device cybersecurity states without increasing the volume of information stored and processed in internal resources.
\end{abstract}

Keywords - cybersecurity monitoring, nodes, and devices of the Internet of things, network infrastructure.

DOI: 10.18421/TEM104-55

https://doi.org/10.18421/TEM104-55

Corresponding author: M.E. Sukhoparov,

St. Petersburg Federal Research Center of the Russian Academy of Sciences (SPC RAS), 39, 14th Line V.O., 199178, Saint-Petersburg, Russian Federation.

Email: isl_box@mail.ru

Received: 14 July 2021.

Revised: 08 November 2021.

Accepted: 15 November 2021.

Published: 26 November 2021.

(c) BY-NC-ND (C) 2021 M.E. Sukhoparov \& I.S. Lebedev; published by UIKTEN. This work is licensed under the Creative Commons Attribution-NonCommercial-NoDerivs 4.0 License.

The article is published with Open Access at www.temjournal.com

\section{Introduction}

The cutting-edge IoT presents the concept of organizing and building a computing network of physical objects interacting with each other and the external environment. The use of technologies for data identification, transmission, and processing necessitates the creation of various cybersecurity state monitoring systems aimed at neutralizing threats to the IoT.

An integrated approach is used to organize the protection of distributed information and telecommunication systems. The implementation of monitoring the CS state of devices, systems, and networks requires interaction control, ensuring communication security, and the protection of devices [1], [2]. However, cybersecurity state monitoring assumes dynamic development and the emergence of situations requiring analysis when old and new devices and different versions of hardware and software solutions are used simultaneously, and various interface protocols, data processing analysis, and transmission technologies are applied [3].

IoT devices are usually located outside the controlled area. This location requires control when updating software, and optimizing a number of operational processes during the life cycle. Built-in protection systems are not always effective since potential intruders usually have no difficulty in accessing and can search for vulnerabilities on typical devices [4].

When developing information systems, it is necessary to improve the protection, control, and monitoring of IoT device cybersecurity [5], [6]. One of the research trends is associated with the use of information obtained through side channels. Data can be used for this that is not directly related to ongoing processes and calculated during processing, for example, acoustic, electromagnetic, and other radiation. This data provides additional information about the process manifestation [7], [8], which can be considered when analyzing the states of devices.

Many IoT elements possess relatively small hardware and software capabilities, have a limited set of commands with predefined actions and reactions. 
Their functionality does not change during the life cycle. They carry out a programmed sequence of actions when commands are received, and external events occur. IoT devices have a limited number of analyzed functional parameters. This fact allows machine learning methods to identify a normal (safe) state in which predefined processes are performed, and an abnormal (dangerous) state in which parameter and characteristic values appear to indicate deviations from the norm. The research purpose is to develop an approach to identifying the cybersecurity state of IoT devices using data sampling segmentation based on processed time series recorded from probes and sensors during various processes.

\section{Problem Statement}

IoT devices are "black boxes" in which software and hardware from various companies can be used. As a result, the source code is not always known and may contain undocumented features. A large number of IoT devices do not allow for hardware and software modification. Built-in protection systems are implemented, and in the case of integrity violations, the device can stop functioning. During operation, it becomes necessary to set up, configure, and improve the telecommunications information and system characteristics. The unification of elements of IoT devices and their typing allows a potential intruder to investigate and search for vulnerabilities at the software and hardware levels. The application of wireless communication technologies makes it possible to implement attempts to transmit control commands to introduce compromised devices into the network to carry out destructive influences. However, assessing the CS state requires assessing a large number of parameters and situations in which their deviation occurs, which requires adaptation and the development of protective mechanisms. In this regard, it becomes necessary to analyze several internal and side information channels simultaneously.

IoT devices constantly interact with each other. This interaction causes simultaneous changes in many parameters. Therefore, it is necessary to analyze several preceding discrete states for cybersecurity state identification.

The proposed approach is based on the use of patterns. A behavioral pattern is formed by numerous time-synchronized sequences of values of time series of parameters of processes occurring within devices from internal and external sensors. The behavioral pattern contains information about the functioning of the internal processes in IoT devices. It stores synchronized data on resource loading and consumption, and statistical information on various parameters [8], [9].

Measuring devices and sensors record the ongoing process in IoT devices. They give out numerical sequences. The tuples of characteristic values from various control elements are synchronized at discrete times.

The formal statement of the problem of identifying the IoT device cybersecurity state can be represented as follows: $Z$ is a set of the device states, and $C$ is a set of classes of states containing dangerous $C_{l}$ and safe $C_{2}$ states. In a safe state, predefined processes run. Dangerous conditions are determined by the value deviations of the indicators and their groups from the expected values.

There is a finite training set of known states $\left\{z_{1}, \ldots, z_{l}\right\} \in Z$. The subsets of classes $\left\{C_{1}, C_{2}\right\} \in C$ are associated with this set. It is necessary to find an algorithm that reflects a set into a set [10], [11].

The processing of data coming from devices enables the identification of states [12], [13].

Identification processes are based on machine learning methods. The training sample is processed using the classifying algorithm. The tuples of timeseries values $x_{1}, x_{2}, \ldots, x_{n}$ reflect the process behavior [14].

Various external and internal factors affect IoT devices during their operation. These factors can include commands, events of reception, transmission, and information processing. As a result, the ranges of the registered parameters may be mixed.

Let $V$ be a set of factors influencing the values of features. The set $X$ is characterized by a set of feature tuples. Figure 1. shows training sample that takes the form of the labeled sample $\left\{v_{j},\left\{\left(x_{i}, c_{i}\right)\right\}_{i=1}^{N}\right\}_{j=1}^{M}$, where $N$ is the amount, and $M$ is the number of factors affecting the sample values.

\begin{tabular}{|c|c|c|c|c|c|c|}
\hline \multirow{2}{*}{$\begin{array}{c}\text { Influencing } \\
\text { factor }\end{array}$} & $\begin{array}{c}\text { Class mark } \\
\text { (event }\end{array}$ & \multirow{2}{*}{$\begin{array}{c}\text { Time } \\
\text { labelling) }\end{array}$} & \multicolumn{4}{|c|}{ Feature values } \\
\cline { 3 - 7 } & $c_{0}$ & $t_{0}$ & $x_{10}$ & $f_{2}$ & $\ldots$ & $f_{1}$ \\
\hline \multirow{5}{*}{$v_{1}$} & $c_{1}$ & $t_{1}$ & $x_{11}$ & $x_{21}$ & $\ldots$ & $x_{n 0}$ \\
\cline { 2 - 7 } & $c_{0}$ & $t_{2}$ & $x_{12}$ & $x_{22}$ & $\ldots$ & $x_{n 1}$ \\
\cline { 2 - 7 } & $c_{0}$ & $t_{3}$ & $x_{10}$ & $x_{20}$ & $\ldots$ & $x_{n 0}$ \\
\cline { 2 - 7 } & $c_{0}$ & $t_{4}$ & $x_{11}$ & $x_{21}$ & $\ldots$ & $x_{n 1}$ \\
\hline \multirow{4}{*}{$v_{1}$} & $c_{1}$ & $t_{5}$ & $x_{12}$ & $x_{22}$ & $\ldots$ & $x_{n 2}$ \\
\cline { 2 - 7 } & $c_{1}$ & $t_{6}$ & $x_{10}$ & $x_{20}$ & $\ldots$ & $x_{n 0}$ \\
\cline { 2 - 7 } & $c_{0}$ & $t_{7}$ & $x_{11}$ & $x_{21}$ & $\ldots$ & $x_{n 1}$ \\
\cline { 2 - 7 } & $c_{1}$ & $t_{8}$ & $x_{12}$ & $x_{22}$ & $\ldots$ & $x_{n 2}$ \\
\hline$\ldots$ & $\ldots$ & $\ldots$ & \multicolumn{5}{|c}{$\ldots$} \\
\hline$v_{k}$ & $c_{1}$ & $t_{m}$ & $x_{1 m}$ & $x_{2 m}$ & $\ldots$ & $x_{n m}$ \\
\hline
\end{tabular}

Figure 1. Presentation of data from an IoT device 
It is necessary to construct a classifying algorithm that considers the influence of factor $v, \alpha: X \stackrel{V}{\longrightarrow} C$.

Thus, the purpose is to process information from the side and internal sources and identify the device CS state based on this information in situations where various factors influence.

By applying information about the acting factors $v_{l}, v_{2}, \ldots, v_{k}$, it is possible to split the training sample into segments, where factor $v_{i}$ had acted:

$$
X_{1} U X_{2} U \ldots U X_{k}=X u X_{j} \cap X_{i} \neq 0 \quad \forall i \neq j \text {. }
$$

The classification algorithms $a_{r}, r=1, \ldots, k$ are given their own segments. The probability of belonging to a class under the influence of a known factor $v_{r}$, entering the input of a tuple $x$ is determined as:

$$
a_{r}(x)=\max _{j=1.2} P_{r}\left(C_{j} \mid x\right)
$$

The resulting class predicted by the classifier ensemble for tuple $x$ can be determined using the values of the functions $F_{1}$ and $F_{2}$ :

$$
\begin{array}{r}
F_{1}\left(a_{1}(x), \ldots, a_{k}(\mathrm{x})\right)= \\
\frac{1}{k} \sum_{r=1}^{k} a_{r}(\mathrm{x}) F_{2}\left(a_{1}(x), \ldots, a_{k}(\mathrm{x})\right)=1- \\
F_{1}\left(a_{1}(\mathrm{x}), \ldots, a_{k}(\mathrm{x})\right.
\end{array}
$$

The implementation of the ensemble of basic algorithms is described by the expression:

$$
\begin{array}{r}
a(x)= \\
\varphi\left(F_{1}\left(a_{1}(x), \ldots, a_{k}(x)\right), F_{2}\left(a_{1}(x), \ldots, a_{k}(x)\right)\right) \quad(3)
\end{array}
$$

where $\varphi$ is a decision rule enabling to determine the probabilistic estimate and establish the class number.

The proposed approach to identifying an IoT device cybersecurity state is distinguished by the use of template sequences containing data from the past conditions of the device operation. Template sequences contain synchronized time series of numeric values derived from recording elements during the process execution.

The application of the proposed approach assumes the device "tuning" to the predetermined operating modes at the initial stage. Data preprocessing is performed using classification algorithms and involves the computation of threshold values. At the stage of functioning, the current state is correlated with that determined at the initial stage.

Further, deviations are analyzed using trained classifiers.

\section{Simulation Experiment Results}

Detection of changes in the computational environment is an urgent problematic issue for cybersecurity state monitoring. In this regard, it becomes necessary to identify the state of a functioning device as safe or unsafe.

The purpose of the experiment was to identify the computational node state, determined by the data processing algorithm, based on the digitized indicators of a load of computing resources [15], [16]. Time-synchronized percentages of the system load monitor were identified as a behavioral patternforming sequence [17].

The processor and memory load indicators were taken from the system monitor on a functioning computational node. The following processes were chosen as factors $v_{1}, v_{2}, v_{3}$ influencing the values of the target variables: $v_{1}-$ a file copying, $v_{2}-$ searching for a predetermined information template, $v_{3}-$ calculating and adding additional information to the file. The actions of the processes determined the states of the nodes $Z_{1}, Z_{2}, Z_{3}$.

The second part of the experiment was carried out under conditions when "additional" software was launched on the node in states $Z_{1}, Z_{2}, Z_{3}$, which consumes the processor (CPU) and memory resources. Conditionally assuming that there are no additional processes that take resources in a safe state and that they are present in an unsafe state, the identification of the CS state was determined through the processed time series.

Time-synchronized sequences of CPU and memory resource usage values are represented as output sequences.

A time-series sample for CS states was obtained during the experiment. This sample was divided into training and test samples. Figures 2. and Figure 3. show two-dimensional time-series tuples were supplied to each classification algorithm.

The state determination process consisted of calculating values based on the input sequence data, which were compared by classification algorithms.

The following algorithms were chosen to assess the impact of using subsets on the quality of the results of machine learning models: naive Bayes classifier (NB), linear discriminant analysis (LD), classification trees (CT), and nearest neighbors (KNN).

Figure 4. shows the classification areas obtained from state processing. 

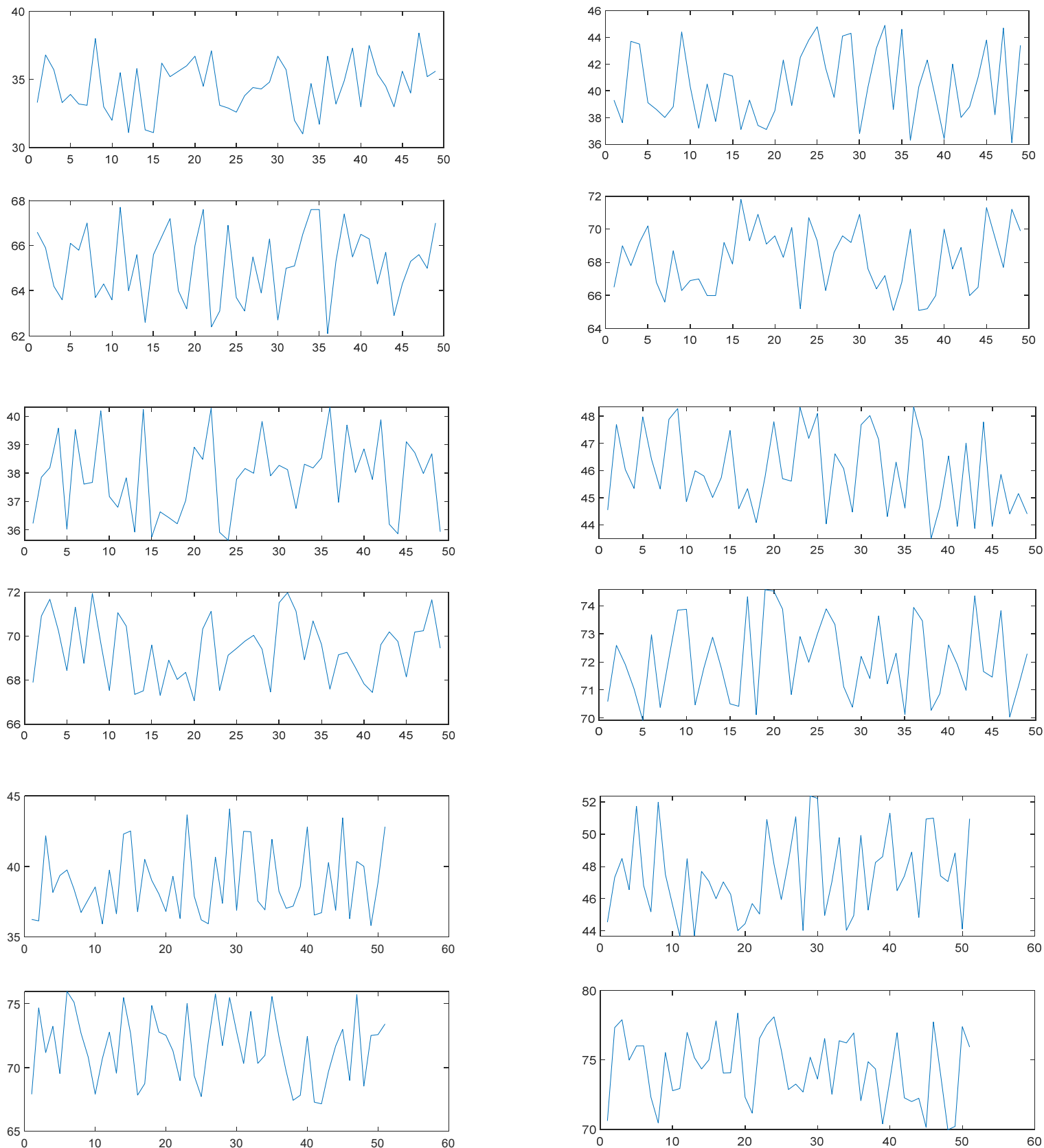

Figure 2. An example of the resource loading percentage sampling (from top to bottom for each pair - memory, processor) from time counts for conditionally normal (left) and abnormal (right) states $Z_{1}, Z_{2}, Z_{3}$ (top down)
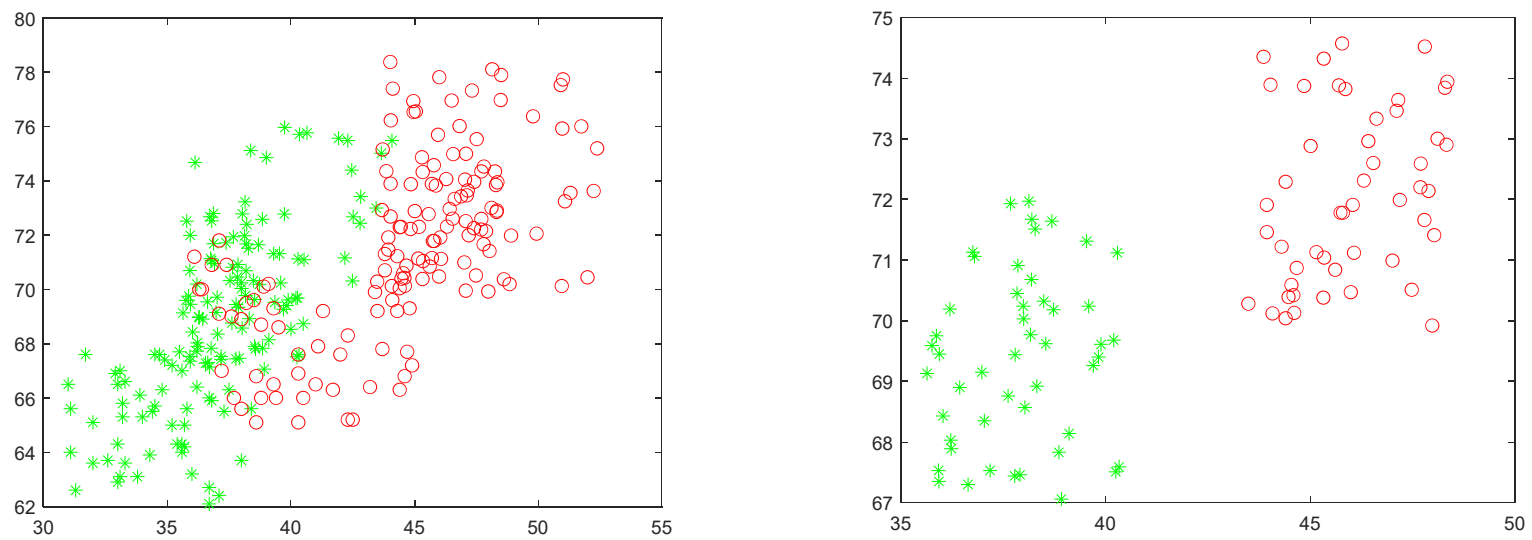

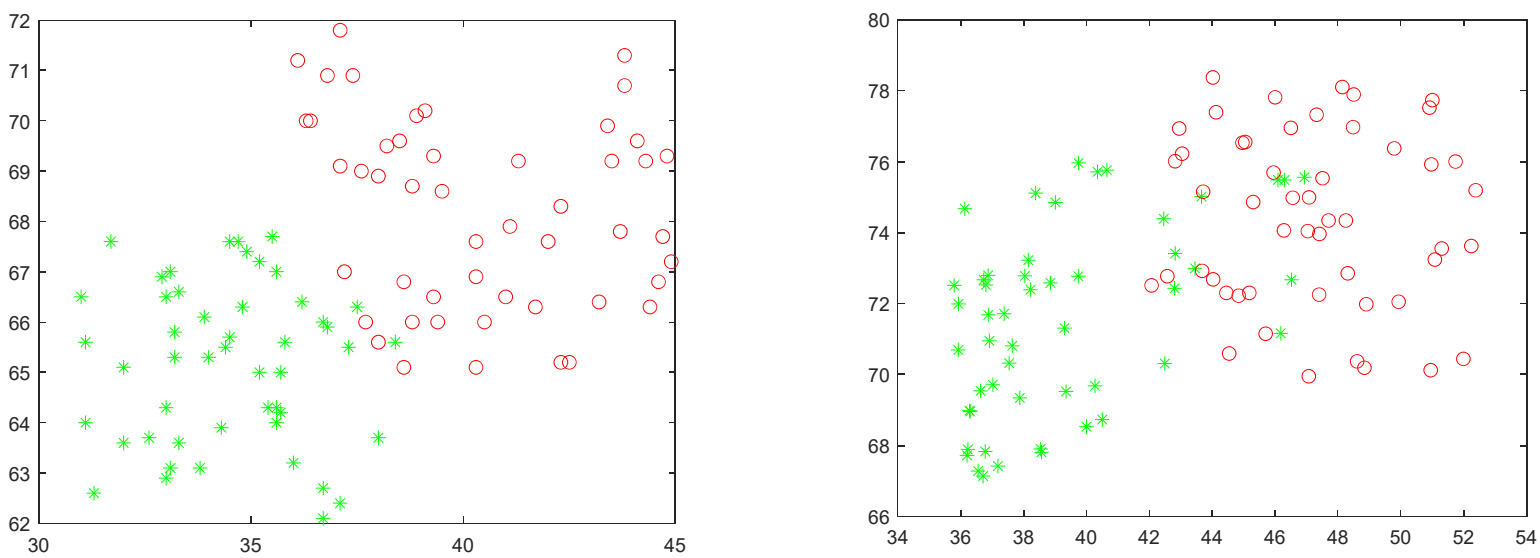

Figure 3. An example of data sampling for values of normal (red) and abnormal (blue) "memory-processor" loading percentage for the entire set (top left) and for each state $Z_{1}, Z_{2}, Z_{3}$
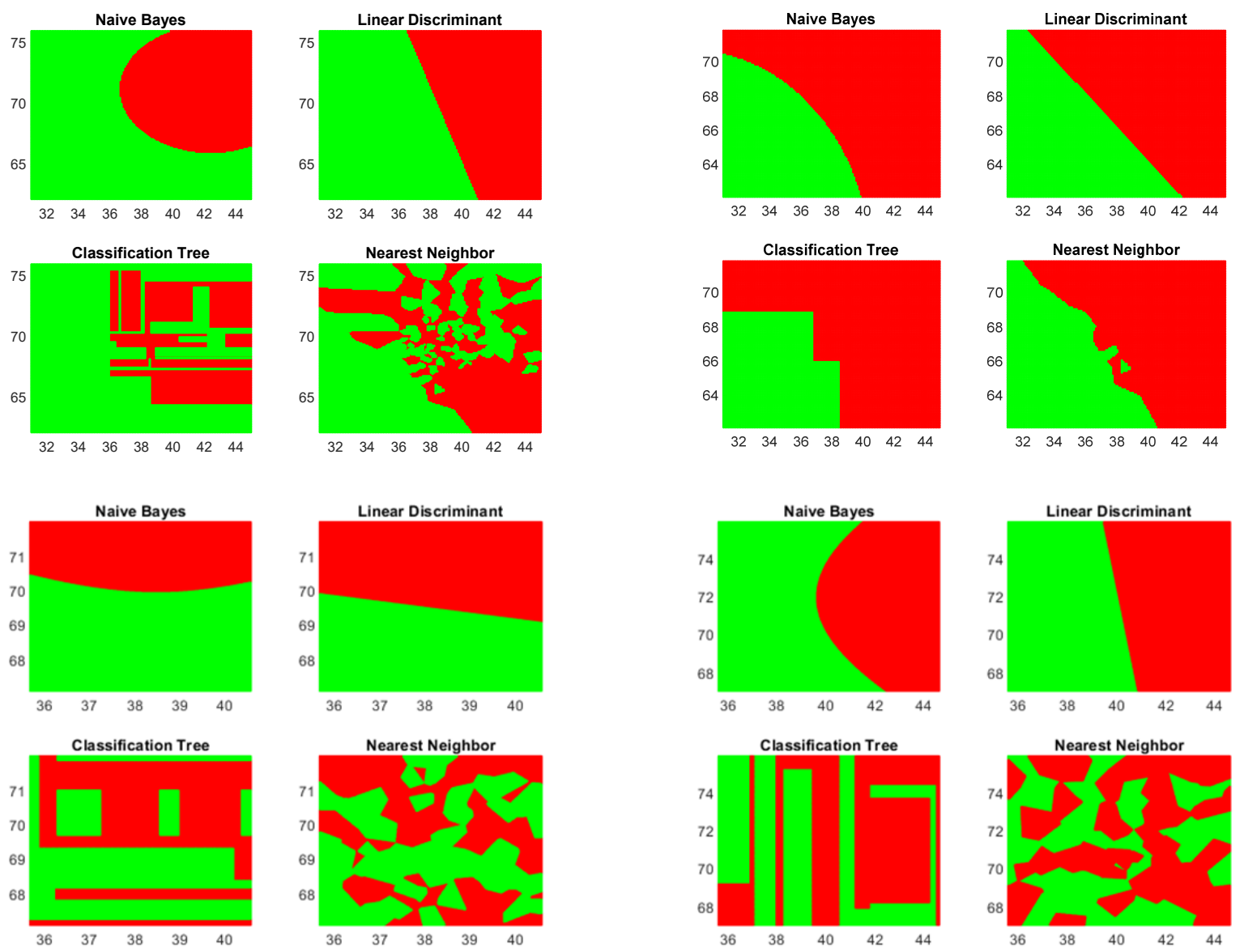

Figure 4. The results of the class region selection for the entire set (top left) and for each state $Z_{1}, Z_{2}, Z_{3}$

Table 1. shows the results of testing the classifiers.

Table 1. The results of classification algorithms

\begin{tabular}{|cccccccc|}
\hline Model & \multicolumn{3}{c}{ The entire sample X } & \multicolumn{3}{c|}{ Mean value } \\
& XU & $+X_{2}+\mathbf{X}_{\mathbf{3}}$ \\
NB & 0.77 & 0.72 & 0.76 & 0.79 & 0.75 & 0.78 \\
LD & 0.77 & 0.71 & 0.76 & 0.78 & 0.75 & 0.78 \\
CT & 0.91 & 0.81 & 0.84 & 0.92 & 0.81 & 0.87 \\
KNN & 0.96 & 0.83 & 0.90 & 0.98 & 0.86 & 0.95 \\
Ensemble & 0.98 & 0.94 & 0.93 & 0.99 & 0.97 & 0.98 \\
\hline
\end{tabular}

These results show that the use of classifiers on selected subsets can improve the classification quality indicators.

Thus, the proposed approach makes it possible to determine the class of the current state. When the data sample segmentation allows reducing the size of the overlapping areas of classes, it is possible to improve the classification quality indicators. 


\section{Conclusion}

The process of monitoring the cybersecurity state of IoT devices is laborious and requires the analysis of numerous indicators. The achievement of the specified qualitative indicators for determining CS states depends on the properties of the analyzed data. Both insufficient and excessive numbers of informative indicators can lead to identification errors.

The proposed approach to identifying the CS state of an IoT device based on machine learning methods makes it possible to determine the current state. It is executed by preliminary processing of time-series information received from recording devices in various operating modes. The presence of predetermined training samples enables the exclusion of time-consuming processes for calculating parameters of operating modes. The automatic determination of characteristics and the use of the dataset segmentation allow for determining the current state class without significant expenditure of computational resources.

The need to synchronize time series from different sources is the main limitation of the proposed approach. Another limitation affecting the qualitative indicators of the method application is the selection of the lengths of considered intervals. The correct formation of the training sample has a significant impact on further results. It is necessary to analyze possible correlations of variables and the presence of a sufficient number of classified sequences to improve the quality. Such problematic issues can be partially addressed by increasing the sample size. Combining characteristics can improve the accuracy of assessing the device state, but this process requires additional research. The organization of external data centers to which IoT devices can send information about their functioning for further analysis of the state is another direction in developing such solutions, regarding the relatively limited resources of IoT devices.

The main advantage of the proposed approach is the relative simplicity of its implementation. The proposed approach to identifying the cybersecurity state of IoT devices differs from the known ones by using information about influencing factors that allows for segmenting data samples and processing them separately.

\section{References}

[1]. Yeung, D. Y., \& Ding, Y. (2003). Host-based intrusion detection using dynamic and static behavioral models. Pattern recognition, 36(1), 229243.

[2]. Igure, V. M., Laughter, S. A., \& Williams, R. D. (2006). Security issues in SCADA networks. computers \& security, 25(7), 498-506. https://doi.org/10.1016/j.cose.2006.03.001.

[3]. Sukhoparov, M. E., \& Lebedev, I. S. (2020) Identification the Cybersecurity State for the Internet of Things Devices in Information and Telecommunication Systems. Systems of Control, Communication and Security, 3, 252-268. https://doi.org/10.24411/2410-9916-2020-10310 (in Russian).

[4]. Vyas, A., \& Batra, U. (2020) Identification of Safety and Security Vulnerabilities in Cyber Physical Systems. In: Batra U., Roy N., Panda B. (eds) Data Science and Analytics. REDSET 2019. Communications in Computer and Information Science, vol 1230, 3-13. Springer, Singapore. https://doi.org/10.1007/978-981-15-5830-6 1.

[5]. Schlimmer, J. C., \& Granger, R. H. (1986). Incremental learning from noisy data.Machine learning, 1(3), 317-354.

https://doi.org/10.1023/A:1022810614389.

[6]. Khan, S., \& Yairi, T. (2018). A review on the application of deep learning in system health management. Mechanical Systems and Signal Processing, 107, 241-265. https://doi.org/10.1016/j.ymssp.2017.11.024.

[7]. Bevir, M. K., Osullivan, V. T., \& Wyatt, D. G. (1981). Computation of electromagnetic flowmeter characteristics from magnetic field data. Journal of Physics D Applied Physics, 14(3), 373-388.

[8]. Sethi, T. S., \& Kantardzic, M. (2018). Handling adversarial concept drift in streaming data. Expert systems with applications, 97, 18-40. http://doi.org/10.1016/j.eswa.2017.12.022.

[9]. Khan, S., \& Yairi, T. (2018). A review on the application of deep learning in system health management. Mechanical Systems and Signal Processing, 107, 241-265. https://doi.org/10.1016/j.ymssp.2017.11.024.

[10]. Tsai, S. Y., \& Chang, J. Y. (2018, February). Parametric study and design of deep learning on leveling system for smart manufacturing. In 2018 IEEE International Conference on Smart Manufacturing, Industrial \& Logistics Engineering (SMILE) (pp. 48-52). IEEE. https://doi.org/10.1109/SMILE.2018.8353980.

[11]. Bertino, E., \& Islam, N. (2017). Botnets and internet of things security. Computer, 50(2), 76-79. https://doi.org/10.1109/MC.2017.62.

[12]. Bendat, J. S., \& Piersol, A. G. (1980). Engineering applications of correlation and spectral analysis. New York.

[13]. Zasov, V. A., Tarabardin, M. A., \& Nikonorov, E. N. (2009). Algorithms and Devices for Identifying Input Signals Problems of Control and Diagnosis of Dynamic Objects. Vestnik SSAU, 2(18), 115-122. 
[14]. Lockhart, D. J., Dong, H., Byrne, M. C., Follettie, M. T., Gallo, M. V., Chee, M. S., ... \& Brown, E. L. (1996). Expression monitoring by hybridization to high-density oligonucleotide arrays. Nature biotechnology, 14(13), 1675-1680. https://doi.org/10.1038/nbt1296-1675.

[15]. Golub, T. R., Slonim, D. K., Tamayo, P., Huard, C., Gaasenbeek, M., Mesirov, J. P., ... \& Lander, E. S. (1999). Molecular classification of cancer: class discovery and class prediction by gene expression monitoring. science, 286(5439), 531-537. https://doi.org/10.1126/science.286.5439.531.
[16]. Anderberg, M. R. (1976). Cluster Analysis for 4pplications. New yok Academic, 1973. I2 1 E. Diday and JC Simon, Clustering Analysis: Communicorion: gree. vol. 10.

[17]. Dembele, D., \& Kastner, P. (2003). Fuzzy C-means method for clustering microarray data. bioinformatics, 19(8), 973-980. 